\title{
INFLUENCE OF PHASE ERROR ON $M$-QAM DEMODULATION IN A NON-GAUSSIAN ENVIRONMENT
}

\author{
Huu Tuê Huynh, Paul Fortier \\ The authors are with the Department of Electrical and Computer Engineering \\ Laval University, Ste-Foy, Quebec, G1K 7P4 Canada \\ \{huynh, fortier\}@gel.ulaval.ca
}

\begin{abstract}
This paper considers the influence of a deterministic phase error for coherent $M$-QAM demodulators in a non-Gaussian environment; the latter is modeled as a combination of a white Gaussian process and a filtered Poisson process whose enveloped amplitude obeys the Rayleigh distribution. General results show that when approaching the critical limit, the symbol error rate quickly reaches a plateau having a relatively large width.
\end{abstract}

\section{INTRODUCTION}

Digital communications systems are becoming more and more popular in every day use to the point of being considered just another consumer good. Among digital modulation schemes, $M$-QAM is the most employed because of its bandwidth efficiency [1]. To receive QAM signals, we have to use coherent detection which requires a perfect phase synchronization. In practice, the phase error can never be cancelled. In many research works that analyze the performance of QAM, this phase error is rarely taken into account [2, 3]. Furthermore, in the modern operating environment of digital communication systems, non-Gaussian noise is observed as a dominating factor $[4,5]$. It is then quite natural to look at the influence of these two factors on the behavior of QAM demodulators.

In this paper, the non-Gaussian perturbation is considered as the sum of a white Gaussian noise with spectral density $N_{0} /$ 2 and an impulsive noise modeled as a filtered Poisson process with Rayleigh distribution for the envelope amplitude. This model has been used by several authors, among them Kosmopoulos et al. [2]; however, in their work, they just use a first order approximation and apply the Fourier-Bessel technique to perform numerical computations. In practice, the number of impulses occurring in the detection interval is not small enough to justify the first order approximation [4]. It then becomes important to find a way for a general analysis.

The phase error statistics in the presence of non-Gaussian noise are still not well studied. Some preliminary results have been reported for a first order phase locked loop [6]. In such a situation, it is preferable to analyze the receiver behavior for fixed phase errors and to study the symbol error rate for a practical range of these phase error values, up to the critical phase error for each constellation size.

In a recent work [7], using the characteristic function method, we had obtained an exact analytical expression of the joint probability density function $f_{X Y}(x, y)$ of the in-phase and quadrature outputs of a correlator-receiver, which is optimum for additive white Gaussian noise, using the maximum likelihood criteria. The detection thresholds remain unchanged while the phase error $\theta$ makes the received signal move. These changes can be computed analytically in such a way that the symbol error rate computations can be formulated in terms of $f_{X Y}(x, y)$. We can easily compute the symbol error rate for any situation and the numerical values can be obtained as precisely as desired.

The paper is structured as follows. In Section 2, we briefly present the receiver and noise models. It is shown that the statistics of the output noises (in phase and in quadrature) are circularly symmetric. This property is equivalent to saying that the phase and modulus of the noise vector are statistically independent. The exact expression of the symbol error rate is developed in Section 3. Some typical numerical results for symbol error rates in terms of the phase error $\theta$, the average impulse number $\gamma$, and the ratio $\beta$ of impulse noise power over Gaussian noise power are shown in Section 4, where a brief discussion is also given.

\section{RECEIVER AND NOISE MODELS}

\section{A. QAM receiver}

This scheme of modulation is a combination of two carriers, in phase and in quadrature, each of which is modulated in amplitude by a bit stream obtained from a serial to parallel converter. The transmitted signal is given by

$$
s(t)=\sum_{k} A_{k} a(t-k T) \cos \omega_{c} t-B_{k} a(t-k T) \sin \omega_{c} t
$$

where $a(t)$ is the low-pass shaping pulse and $T$ is the symbol duration. $A_{k}$ and $B_{k}$ correspond to the amplitudes of the modulated signal. Given the fact that $a(t)$ is a low-pass signal, it can be chosen so that $a(t) \cos \omega_{c} t$ and $b(t) \sin \omega_{c} t$ are orthonormal. The signal $s(t)$ can then be represented by a two-dimensional vector whose components are $A_{k}$ and $B_{k}$. The vectors $\left(A_{k}, B_{k}\right)$ correspond to points in the QAM constellation.

At the front end of the receiver (see Fig. 1), noise is added to $s(t)$. The coherent receiver uses two correlators. The output components $y_{1}$ and $y_{2}$ with phase error $\theta$ are given by

$$
y_{1}=\int_{0}^{T} r(t) a(t) \cos \left(\omega_{c} t+\theta\right) d t
$$




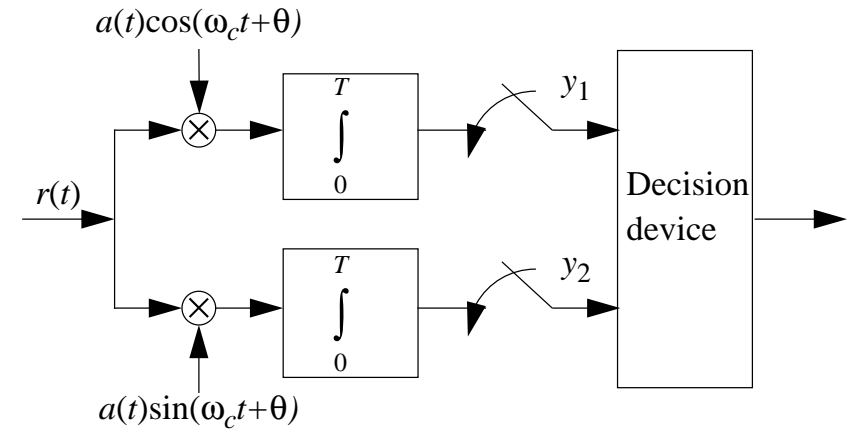

Fig. 1: Correlator receiver with phase error $\theta$.

and

$$
y_{2}=\int_{0}^{T} r(t) a(t) \sin \left(\omega_{c} t+\theta\right) d t
$$

The decision device detects $\left(A_{k}, B_{k}\right)$ based on the observations $\left(y_{1}, y_{2}\right)$.

\section{B. Noise model}

As previously discussed, the additive noise at the front end of the receiver is the sum of two components, the white Gaussian noise with double-sided spectral density $N_{0} / 2$ and the impulsive noise. The Gaussian noise gives as output of the correlators two independent zero-mean Gaussian random variables, $G_{1}$ and $G_{2}$, with variance $\sigma_{G}^{2}=N_{0} / 2$.

Following Bello and Esposito [8], the impulsive noise is modeled as:

$$
i(t)=\sum_{k=1}^{L} C_{k} h\left(t-T_{k}\right) \cos \omega_{c}\left(t-\phi_{k}\right)
$$

where $\phi_{k}$ and $C_{k}$ are respectively the phase and the amplitude of the $k^{\text {th }}$ impulse occurring at instant $T_{k}$. The waveform $h(t)$ is physically considered as the low-pass equivalent RF filter impulse response. It is assumed that the $T_{k}$ 's are independent random variables uniformly distributed over the detection interval $[0, T]$. The number $L$ of impulses occurring during this interval is Poisson distributed with parameter $\gamma$. Let $I_{1}$ and $I_{2}$ be the outputs of the in phase and in quadrature correlators when the input is the impulsive noise process alone. We then have

$$
I_{1}=\sum_{k=1}^{L} P_{k} \cos \left(\phi_{k}+\theta\right)
$$

and

$$
I_{2}=\sum_{k=1}^{L} P_{k} \sin \left(\phi_{k}+\theta\right)
$$

where $P_{k}=\frac{C_{k} H_{k}}{2}$ with $H_{k}$ defined by

$$
H_{k}=\int_{0}^{T} h\left(t-T_{k}\right) a(t) d t
$$

$I_{1}$ and $I_{2}$ are zero mean random variables with the same statistics, of which the variance is noted as $\sigma_{I}^{2}$.

Physically speaking, $P$ can be seen as a random sample of the output envelope when the input is excited by a random impulse occurring in the detection interval.

Finally, the total noises $X$ and $Y$ are given by:

$$
X=G_{1}+I_{1} \text { and } Y=G_{2}+I_{2}
$$

In the following, $X$ and $Y$ will be called the non-Gaussian noise, while $G_{i}$ and $I_{i}$ will be referred to as the Gaussian components and the impulsive components, respectively.

Given the statistical independence of $G_{i}$ and $I_{i}$, the joint characteristic function $\Phi_{X, Y}\left(\omega_{1}, \omega_{2}\right)$ of these random variables can be shown to be only a function of $\rho=\sqrt{\omega_{1}^{2}+\omega_{2}^{2}}$. The joint probability density function of $X$ and $Y$ is given by

$$
f_{X, Y}(x, y)=\int_{0}^{\infty} \rho \Phi_{X, Y}(\rho) J_{0}(r \rho) d \rho
$$

where $J_{0}$ is the Bessel function of order zero and $r$ is then given by

$$
r=\sqrt{x^{2}+y^{2}}
$$

The projection of this non-Gaussian noise over orthonormal axes obtained by any rotation of $x$ and $y$ coordinates will therefore give exactly the same probability density function; this means that phase errors do not affect the statistics of the receiver output noise. This is precisely the circular symmetry property. Consequently, the phase and modulus of the vector $(X, Y)$ are statistically independent. This property allows the use of a bilateral distribution for the amplitude without affecting the validity of the model. In this work we assume that the random envelope sample $P$ follows the bilateral Rayleigh distribution.

$$
f_{p}(\alpha)=\frac{|\alpha|}{\sigma_{p}^{2}} e^{-\frac{\alpha}{\sigma_{p}^{2}}}
$$

These statistics have been successfully used by several authors $[2,9,10]$. They allow us to obtain an exact solution to our problem. These statistics straightforwardly give

$$
f_{X, Y}(x, y)=\frac{e^{-\gamma}}{2 \pi} \sum_{k=0}^{\infty} \frac{\gamma^{k}}{k !} \frac{1}{\sigma_{k}^{2}} \exp \left[\frac{-\left(x^{2}+y^{2}\right)}{2 \sigma_{k}^{2}}\right]
$$

where

$$
\sigma_{k}^{2}=\sigma_{G}^{2}+\frac{k}{\gamma} \sigma_{I}^{2}
$$

In the following, the number of terms used in the numerical computation for symbol error rates is chosen by upper bounding the computational error. The absolute error of these computations due to truncation at $m$ terms can be shown to be 
upper bounded by [7]:

$$
\varepsilon<\frac{\gamma^{m}}{m !}
$$

Researchers often only look at the limit cases of $\gamma$ : very small (severely impulsive noise) or very large (quasi-Gaussian noise). In the first case, they use the first order approximation which means that at most we just have one impulse occurring in the detection interval. In the second case, noise is considered as Gaussian. With (12) there is no more need to use either of these approximations.

\section{INFLUENCE OF PHASE ERROR ON THE SIGNAL SPACE}

Let $M$ and $\theta$ be respectively the size of the constellation and the phase error at demodulation. The detector projects the corrupted received signals on $a(t) \cos \left(\omega_{\mathrm{c}}+\theta\right)$ and $a(t) \sin \left(\omega_{\mathrm{c}}+\theta\right)$. It can be seen that for each $M$, there is a critical phase error beyond which the detector cannot work perfectly, even without noise. This critical phase error is given by

$$
\theta_{c}=\operatorname{tg}^{-1}\left(\frac{1}{2 K-1}\right)
$$

where $K=[\sqrt{M} / 2$ and $\lceil x$ is the smallest integer larger than or equal to $x$.

This phase error rotates the coordinates of the transmitted signals at the output of the correlators, while the statistics of the additive non-Gaussian noise components remain unchanged due to their circular symmetry as previously shown.

Given the symmetry of the constellation and the circular symmetry of the noise vector, it can be shown that the average symbol error probability of the four subsets of the constellation corresponding to the four quadrants is the same. It is then sufficient to look at the first quadrant to compute the average symbol error rate for the whole constellation. Let us index the signals in this subset according to

$$
S_{i j}=\left(X_{i j}, Y_{i j}\right)
$$

where $i$ and $j$ indicate the column and the row corresponding to the position of the signal $S_{i j}$ in the first quadrant constellation as illustrated in Fig. 2 for 16-QAM. Without noise and phase error, the coordinates of $S_{i j}$ are given by:

$$
X_{i j}=(2 i-1) d, \quad\left(Y_{i j}=(2 j-1) d\right)
$$

The projection of these coordinates on the new rotated axes are

$$
X_{i j}^{r}=X_{i j} \cos \theta+Y_{i j} \sin \theta
$$

and

$$
Y_{i j}^{r}=Y_{i j} \cos \theta-X_{i j} \sin \theta
$$

These are the new coordinates of the signals that will be processed by the receiver.

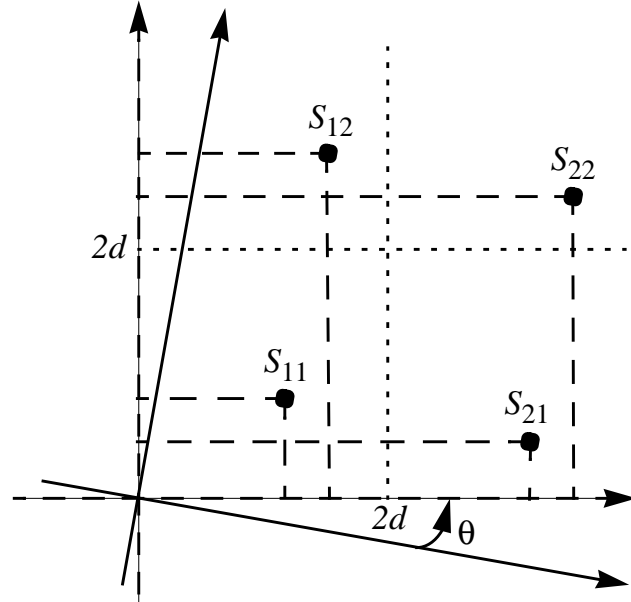

Fig. 2: Rotation of 16-QAM constellation (only the first quadrant is shown).

$$
S_{i j}^{r}=\left(X_{i j}^{r}, Y_{i j}^{r}\right)
$$

In the presence of non-Gaussian noise, these two components are additively corrupted respectively by $(X, Y)$ defined in (8)-(9).

The decision rules remain unchanged, in which the old exact coordinates of the signals are now replaced by the rotated ones. For the points inside the constellation we have:

$$
(2 i-1) d<X_{i j}^{r}+X<2 i d
$$

and

$$
(2 j-1) d<Y_{i j}^{r}+Y<2 j d
$$

The rules are accordingly modified for the boundary points.

With the joint probability density function of $X$ and $Y$ given in (12) the conditional probability of a correct decision can be straightforwardly computed as:

$$
P\left(C \mid S_{i j}\right)=e^{-\gamma} \sum_{k=1}^{\infty} \frac{\gamma^{k}}{k !} P_{k}\left(C \mid S_{i j}\right)
$$

where each term of the right hand series, for the inside points of the constellation, is given by

$$
\begin{aligned}
& P_{k}\left(C \mid S_{i j}\right)= \\
& \quad \frac{1}{4}\left[\operatorname{erf}\left(\frac{2 i d-X_{i j}^{r}}{\sqrt{2} \sigma_{k}}\right)+\operatorname{erf}\left(\frac{X_{i j}^{r}-(2 i-1) d}{\sqrt{2} \sigma_{k}}\right)\right] \\
& \quad \times\left[\operatorname{erf}\left(\frac{2 j d-Y_{i j}^{r}}{\sqrt{2} \sigma_{k}}\right)+\operatorname{erf}\left(\frac{Y_{i j}^{r}-(2 j-1) d}{\sqrt{2} \sigma_{k}}\right)\right]
\end{aligned}
$$

For the vertical and horizontal boundary points we have, respectively 


$$
\begin{aligned}
& P_{k}\left(C \mid S_{K, j \neq K}\right)= \\
& \frac{1}{4}\left[\operatorname{erf}\left(\frac{2 j d-Y_{K j}^{r}}{\sqrt{2} \sigma_{k}}\right)+\operatorname{erf}\left(\frac{Y_{K j}^{r}-(2 j-1) d}{\sqrt{2} \sigma_{k}}\right)\right] \\
& \times\left[1+\operatorname{erf}\left(\frac{X_{K j}^{r}-(2 K-1) d}{\sqrt{2} \sigma_{k}}\right)\right] \\
& P_{k}\left(C \mid S_{i \neq K, K}\right)= \\
& \frac{1}{4}\left[\operatorname{erf}\left(\frac{2 i d-X_{i K}^{r}}{\sqrt{2} \sigma_{k}}\right)+\operatorname{erf}\left(\frac{X_{i K}^{r}-(2 i-1) d}{\sqrt{2} \sigma_{k}}\right)\right] \\
& \times\left[1+\operatorname{erf}\left(\frac{Y_{i K}^{r}-(2 K-1) d}{\sqrt{2} \sigma_{k}}\right)\right]
\end{aligned}
$$

while, for the corner point, we have

$$
\begin{aligned}
& P_{k}\left(C \mid S_{K, K}\right)= \\
& \quad \frac{1}{4}\left[1+\operatorname{erf}\left(\frac{X_{K K}^{r}-(2 K-1) d}{\sqrt{2} \sigma_{k}}\right)\right] \\
& \quad \times\left[1+\operatorname{erf}\left(\frac{Y_{K K}^{r}-(2 K-1) d}{\sqrt{2} \sigma_{k}}\right)\right]
\end{aligned}
$$

Finally, the symbol error probability is given by

$$
P_{e}=1-\frac{4}{M} \sum_{i, j} P\left(C \mid S_{i j}\right)
$$

\section{TYPICAL RESULTS AND DISCUSSION}

Given the very large number of possibilities, but with a limited space, only results for QAM-16 are shown and discussed.

Without phase error, it is known that the symbol error rate presents threshold and plateau phenomena when the noise is relatively impulsive, i.e. small $\gamma$ and $\beta / \gamma » 1$ [7].

Fig. 3 shows the symbol error rate vs. SNR for a typical case with phase error, corresponding to a highly impulsive noise, with $\gamma=0.01$ and $\beta=1$. In this case, the threshold and plateau phenomena still happen in the presence of phase error, as clearly observed.

Fig. 4 shows the SNR penalty with respect to the case of AWGN without phase error when $P_{e}=10^{-4}$ for $\gamma=0.01$ and different values of $\beta$. Normally, the penalty is very high when the noise is very impulsive. Furthermore, when the phase error increases, this penalty increases and rapidly grows when the phase error approaches its critical value. For $\beta=0.01$, the performance is identical to the purely additive white Gaussian noise with the same phase error. In fact, in this situation, $\beta / \gamma=$ 1 ; it has been shown that in such a condition, the influence of the impulsive component is negligeable [7].

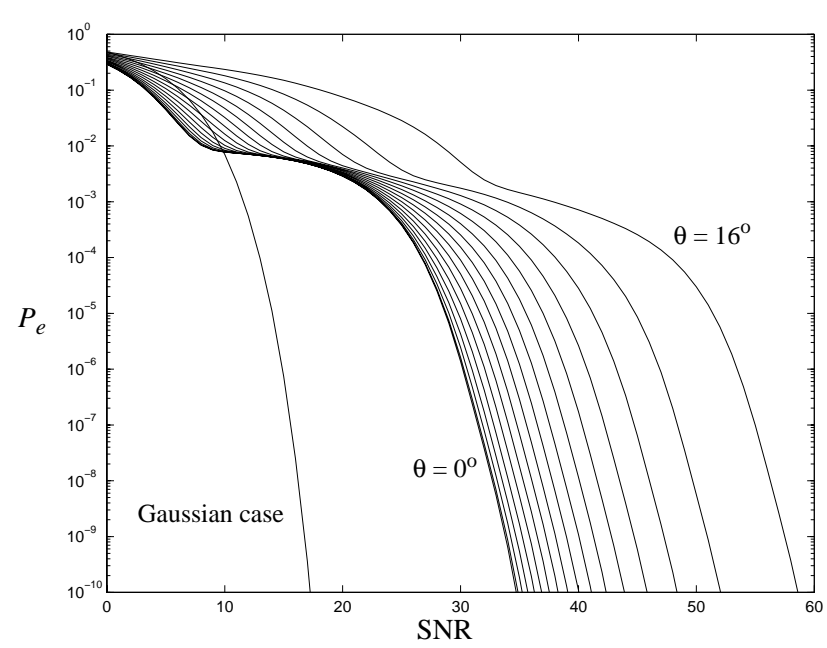

Fig. 3: $P_{e}$ vs. SNR for $\gamma=0.01$ and $\beta=1$.

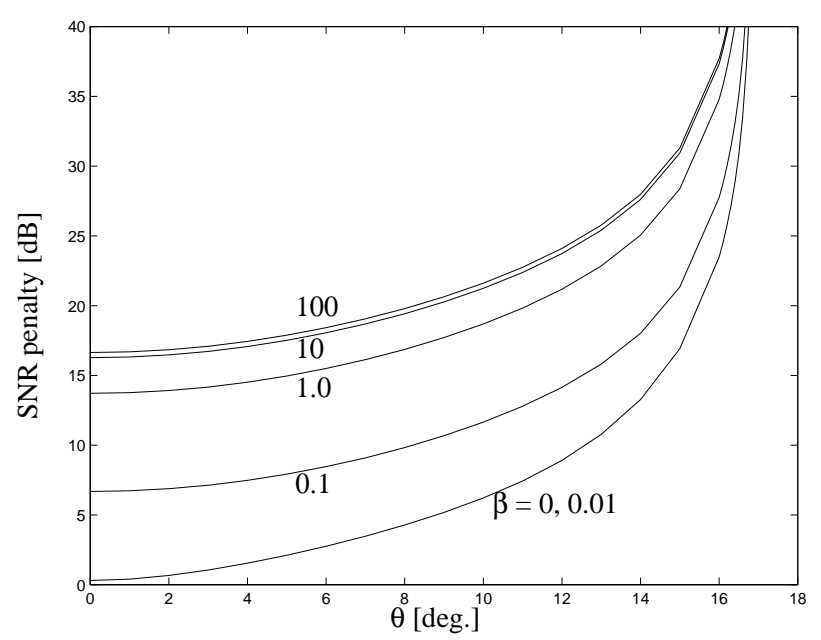

Fig. 4: SNR penalty with respect to AWGN (without phase error) when $P_{e}=10^{-4}$ for $\gamma=0.01$.

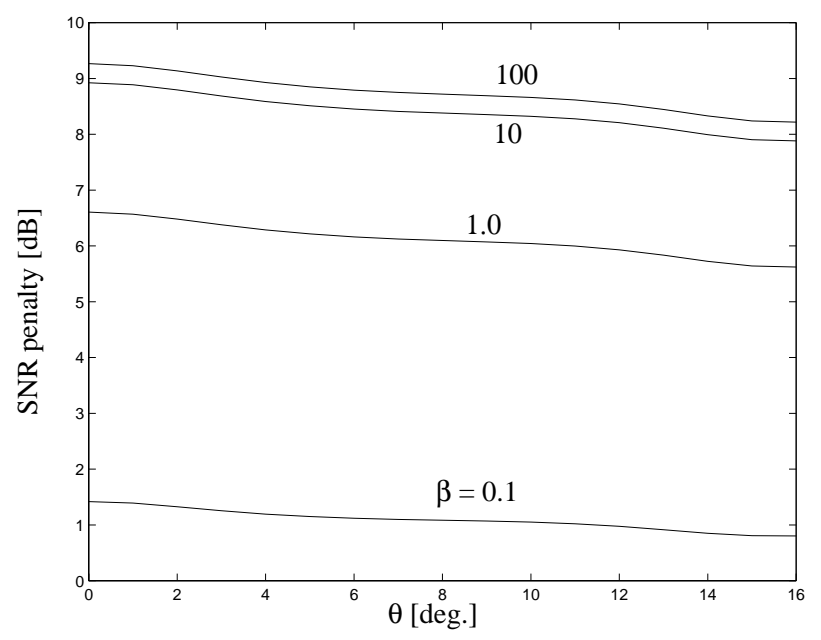

Fig. 5: SNR penalty with respect to AWGN (with phase error) when $P_{e}=10^{-4}$ for $\gamma=0.1$. 


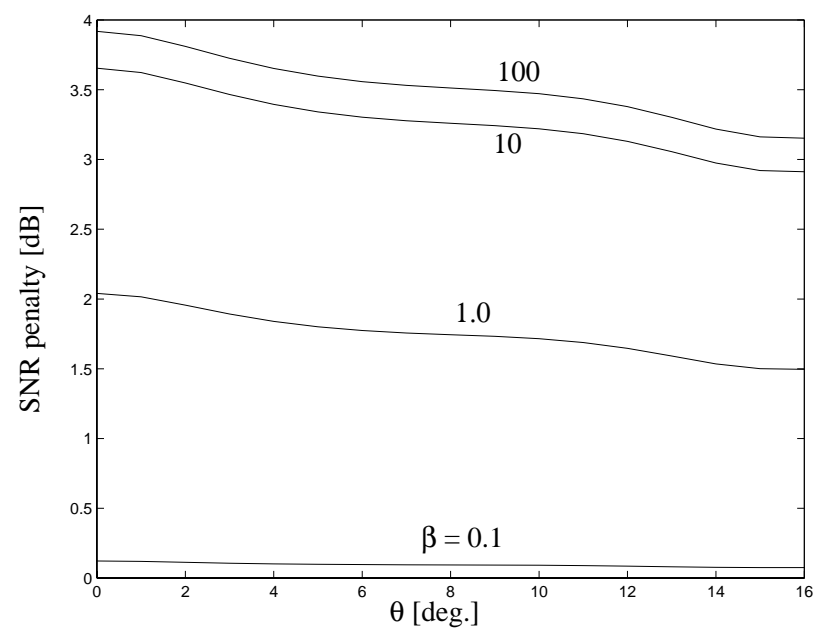

Fig. 6: SNR penalty with respect to AWGN (with phase error) when $P_{e}=10^{-4}$ for $\gamma=1.0$.

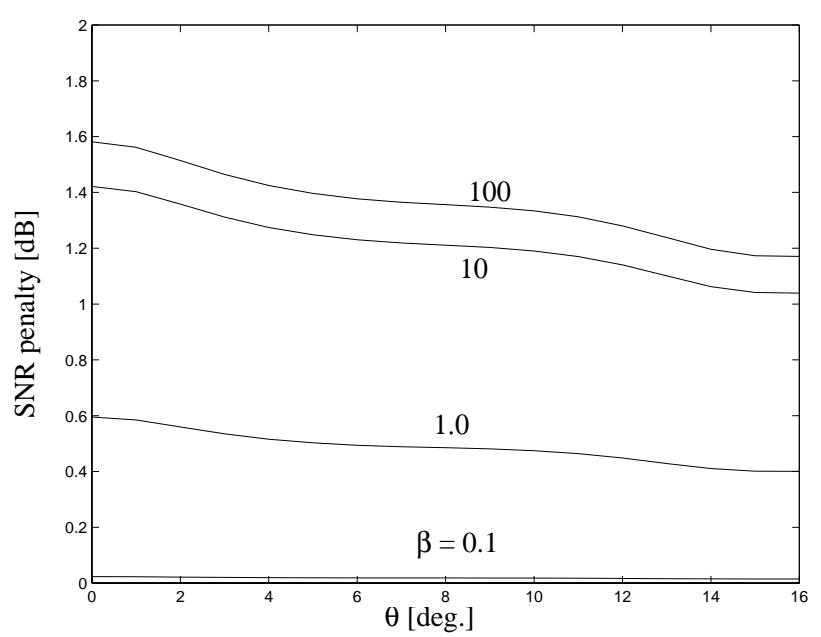

Fig. 7: SNR penalty with respect to AWGN (with phase error) when $P_{e}=10^{-4}$ for $\gamma=5.0$.

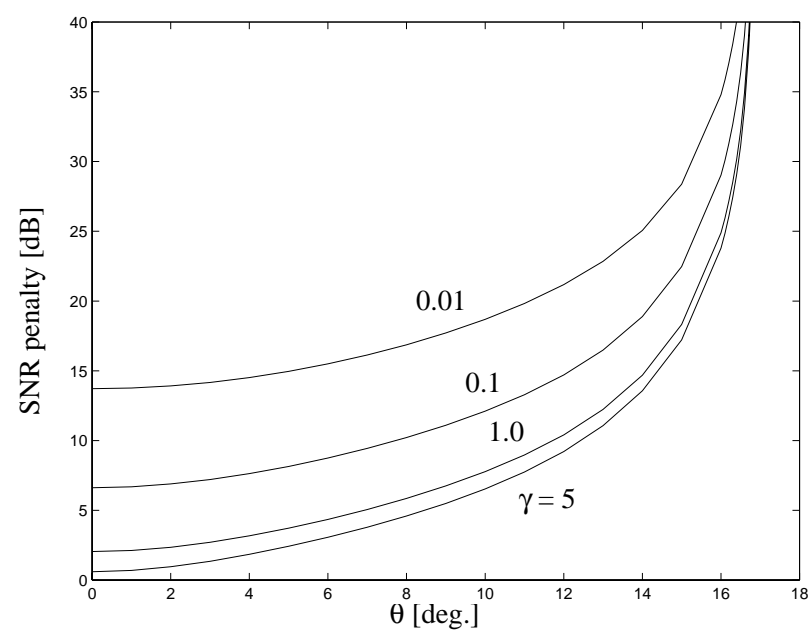

Fig. 8: SNR penalty with respect to AWGN (without phase error) when $P_{e}=10^{-4}$ for $\beta=1.0$.
Figures 5, 6 and 7 show the SNR penalties with respect to AWGN with phase error for various values of $\gamma$ and $\beta$. It is observed that this relative penalty slightly decreases when the phase error increases. Within the range from $0^{\circ}$ to $16^{\circ}$ phase error, for any pair of fixed values of $\gamma$ and $\beta$, the fluctuation of these relative penalties remains around $1 \mathrm{~dB}$. Furthermore, it increases when $\beta$ increases (noise becoming more impulsive) and decreases when $\gamma$ increases (noise approaching the Gaussian case).

We observe that for the values of $\gamma$ considered $(0.01,0.1$, $1.0,5.0)$, when the power of the impulsive component is about equal to the power of the Gaussian component, the overall effect of the non-Gaussian noise rapidly approaches the effect of the impulsive noise alone. However, this penalty strongly depends on $\gamma$, as shown in Fig. 8 .

Although not shown, these behaviors are generally observed for constellations of any size as far as $\gamma$ is larger than the fixed error rate for which we computed the penalty. In cases where $\gamma$ is smaller than this fixed error rate, this SNR penalty could be negative for small phase errors as indicated in Fig. 3.

\section{REFERENCES}

[1] W. T. Webb, L. Hanzo, Modern Quadrature Amplitude Modulation, IEEE Press, New York, 1994.

[2] S. A. Kosmopoulos et al, "Fourier-Bessel Error Performance Analysis and Evaluation of M-ary QAM Schemes in an Impulsive Noise Environment", IEEE Transactions on Communications, vol. 39, No. 3, p. 398, March 1991.

[3] J. S. Seo, S. J. Sho, K. Feher, "The performance of multiary QAM systems in the presence of non-gaussian impulsive noise", in Proc. ICC'87, Toronto, Ont., Canada, pp. 18321836.

[4] N. Morinaga, "Research activities on radio communications in Osaka University", First Kansaï-Québec Workshop on Wireless Personal Communications, Montreal, October 18-19, 1994.

[5] E. N. Skomal, Man-Made Radio Noise, Van Nostrand Reinhold Company, New York, 1978.

[6] J. E. Ohlson, "Phase-Locked Loop Operation in the Presence of Impulsive and Gaussian Noise, IEEE Transactions on Communication, vol. COM-21, no. 9, September 1973, pp. 991-996.

[7] H. T. Huynh, B. Vo, P. Fortier, "Performance of M-QAM in a Non-Gaussian Environment", International Journal of Electronics and Communications, vol. 51, no. 5, pp. 255-262, September 1997.

[8] P. A. Bello and R. Esposito, “A new method for calculating probabilities of errors due to impulsive noise", IEEE Trans. Comm. Technol., vol. COM-17, p. 368, June 1969.

[9] H. T. Huynh, "C-PSK Performance in the Presence of Impulsive Noise: A Simulation Study", International Journal of Electronics and Communications, vol. 34, No. 5, pp. 196, 1980.

[10] D. Middleton, "Statistical-physical models of electromagnetic interference", IEEE Trans. Electromag. Compat., vol. EMC-19, August 1977. 